\title{
Frecuencia de úlceras por presión y los factores asociados a su presentación, en pacientes de un hospital nacional de Lima, Perú
}

\author{
Frequency of pressure ulcers and the factors associated with their presentation, in patients of a national
} hospital in Lima, Peru

Yovana Flores-Lara 1,a, Jesús Rojas-Jaimes ${ }^{2, b}$, Jenny Jurado-Rosales ${ }^{3, a}$

\section{RESUMEN}

Objetivos: Determinar la frecuencia de úlceras por presión (UPP) y los factores asociados a su presentación, en pacientes de un hospital nacional de Lima, Perú. Material y métodos: Se utilizaron los datos del número de UPP por semanas epidemiológicas de las salas de hospitalización obtenidos por la Oficina de Inteligencia Sanitaria del Hospital Nacional Edgardo Rebagliati durante el año 2016. Se determinaron las frecuencias según servicio de hospitalización. Se utilizó Social Science Statistics, para determinar Chi Cuadrado. Resultados: Los servicios con mayor frecuencia de UPP fueron UCI $(41,67 \%)$ y cirugía general $(16,67 \%)$, medicina interna $(9,52 \%)$ y obstetricia $(6,94 \%)$ y existió una relación estadística entre la frecuencia de UPP y UCI, cirugía general, medicina interna y obstetricia $(\mathrm{p}<0,005)$. Conclusiones: Se encontró que la frecuencia de úlceras por presión se asoció con el servicio de hospitalización del paciente, siendo más frecuente en la unidad de cuidados intensivos. Más del $70 \%$ de los casos de UPP ocurrieron en la UCI, hospitalización de cirugía, medicina y obstetricia.

PALABRAS CLAVE: Úlceras por presión, factor de riesgo, morbilidad. (Fuente: DeCS BIREME).

\section{SUMMARY}

Objectives: To determine the frequency of pressure ulcers (PU) and factors associated with in patients of a national hospital in Lima, Peru. Methods: Data from the Oficina de Inteligencia Sanitaria del Hospital Nacional Edgardo Rebagliati regarding PU per epidemiologic week during 2016 were gathered. Frequencies by hospitalization service were calculated using Social Science Statistics, Chi squared was used to compare proportions. Results: The services with the highest frequency of PU were the ICU (41.67\%), general surgery (16.67\%), internal medicine $(9.52 \%)$ and obstetrics (6.4\%); a statistical difference was found between the frequency of PU at ICU, general surgery, internal medicine vs. obstetrics $(\mathrm{p}<0.005)$. Conclusions: the frequency of PU correlated with the hospitalization service, being higher in the ICU, general surgery and internal medicine services compared to obstetric services. More than $70 \%$ of PUs occurred at no-obstetric services.

KEYWORDS: Pressure ulcers; risk factor; morbidity. (Source: MeSH NLM).

\footnotetext{
Unidad de Cuidados Intensivos 2C, Hospital Edgardo Rebagliati Martins. Lima, Perú.

Facultad de Salud, Universidad Privada del Norte. Lima, Perú.

Oficina de Inteligencia Sanitaria, Unidad de Epidemiologia, Hospital Edgardo Rebagliati Martins. Lima, Perú.

Licenciada en Enfermería.

Magister en Biología Molecular.
} 


\section{INTRODUCCIÓN}

Uno de los pilares de todo sistema de salud es la prevención de eventos adversos en los pacientes en las fases de pre-hospitalización, emergencia y hospitalización $(1,2)$. En este sentido las ulceras por presión (UPP) representan uno de los problemas que el sistema de salud debe trabajar en especial en la prevención (1). Las UPP son reportadas desde tiempos muy antiguos en los tratados médicos y en algunas momias egipcias se describen este tipo de lesiones (3).

Las UPP representan un problema en Salud Publica extendido y de suma importancia a nivel mundial, la Organización Mundial de la Salud asegura que la frecuencia de UPP, con un promedio de $8,91 \%$ a nivel hospitalario, es un indicador de calidad de los servicios asistenciales y afecta principalmente a los pacientes críticos sin distinción social (4-7). Existe consenso entre las asociaciones científicas, que la prevención es la mejor forma de abordar la problemática de las ulceras por presión. Según un estudio en Suecia el riesgo de presentar una UPP debe ser evaluada hasta las ocho horas del ingreso del paciente al área de hospitalización y de emergencia en las cuales se reportan en una cantidad importante casos y la estancia prolongada incrementa el riesgo de aparición de una UPP, sumado además que en emergencia se descuida la prevención de las ulceras por lo que se recomienda un informe sobre el riesgo de las UPP en las áreas de emergencia y hospitalización (1).

Los factores de riesgo para el desarrollo de las UPP son: la disminución de la movilidad, desnutrición, bajo índice de masa corporal, disminución de la salud física que incluye factores como la oxigenación y perfusión, edad avanzada, temperatura corporal, fricción, raspado, humedad de la piel, dolor, drogas usadas, algunos tipos de dispositivos médicos, deterioro de la cognición, percepción sensorial y comorbilidades. Además, hay que agregar los factores de riesgo asistenciales como la deficiencia de personal, del conocimiento del grupo asistencial sobre la evaluación y prevención de ulceras, la calidad de la intervención y el uso de guías sobre UPP (8-10).

Por lo expuesto es necesario conocer la frecuencia de UPP en nuestra realidad y cuáles serían los factores de riesgo para desarrollarlas. Con este conocimiento de pondría énfasis en la prevención y tratamiento de las UPP que afectan la morbimortalidad, e incrementa el tiempo y costo hospitalario.
El objetivo del estudio fue determinar la frecuencia de úlceras por presión y los factores asociados a su presentación, en pacientes de un hospital nacional de Lima, Perú.

\section{MATERIAL Y MÉTODOS}

Estudio descriptivo, retrospectivo y relacional, realizado en las salas de hospitalización del Hospital Nacional Edgardo Rebagliati durante el año 2016.

Se colectaron los datos de las UPP según semana epidemiológica, en las salas de hospitalización del Hospital Nacional Edgardo Rebagliati durante el año 2016. La colecta de datos fue realizada por la Oficina de Inteligencia Sanitaria del Hospital Nacional Edgardo Rebagliati como parte de sus funciones rutinarias. Los datos colectados incluyeron edad, genero, diagnóstico, servicio y tiempo de hospitalización del paciente, siendo ingresados en el sistema informático de la Oficina Sanitaria. Además, se revisaron las historias clínicas en los servicios. El criterio de selección fue paciente con presencia de úlcera por presión (UPP) durante el año 2016.

Se utilizó estadística descriptiva, se determinaron frecuencias absolutas y relativas con el programa Excel 2013. Se empleó Chi-Cuadrado para determinar la relación entre las variables, que fueron UPP y servicios de hospitalización, considerando un nivel de significancia del 5\%. Se usó el programa estadístico de acceso libre "Social Science Statistics".

Se protegió la identidad de los datos de los pacientes. El estudio no fue revisado por un Comité de ética.

\section{RESULTADOS}

Se registraron 30 casos de úlceras por presión en el año 2016. Diecinueve $(63,3 \%)$ fueron mujeres y $36,7 \%$, varones. El número de casos por servicio se muestran en el gráfico 1.

La frecuencia de ulceras presión en pacientes por servicio de hospitalización fueron: Medicina Interna $8(9,52 \%)$, Cuidados intensivos $5(41,67 \%)$, Cirugía general $4(16,67 \%)$, Obstetricia $5(6,94 \%)$ y otros 8 $(1,65 \%)(\mathrm{p}=0,004241)$. Como se observó, las úlceras por presión ocurrieron con más frecuencia en los pacientes hospitalizados en cuidados intensivos, en comparación con los otros servicios. 


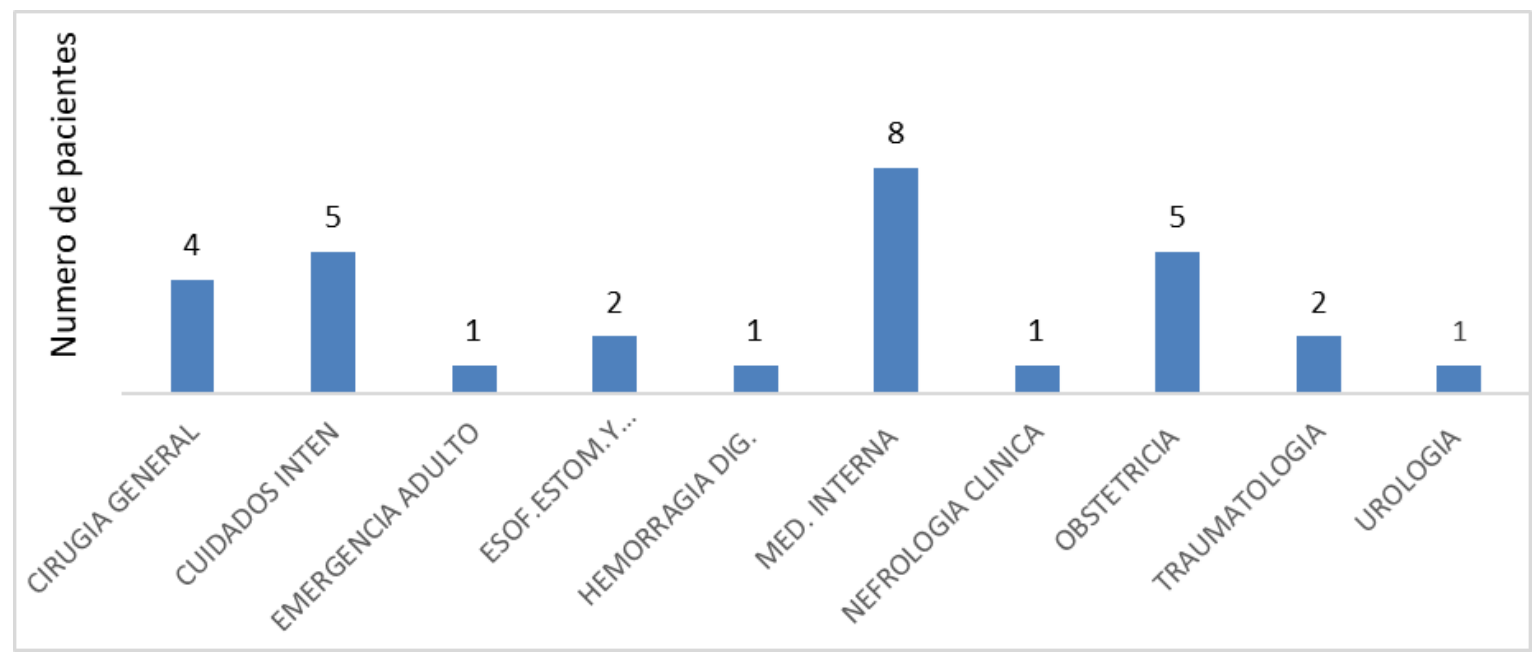

Gráfico 1. Número de pacientes con úlceras por presión (UPP) según áreas de hospitalización.

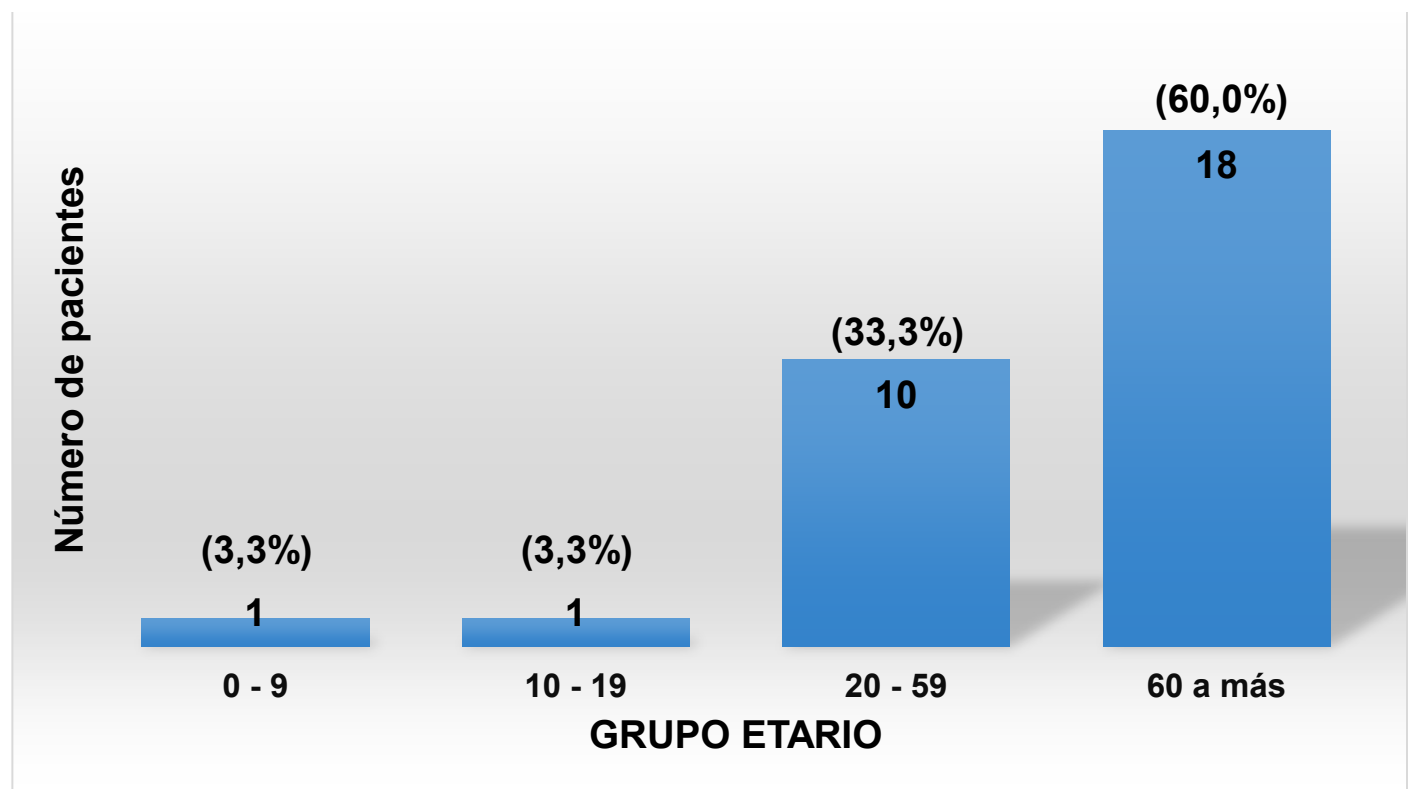

Gráfico 2. Frecuencias relativas porcentuales del número de ulceras por presión (UPP) en pacientes respecto a los grupos etarios.

La frecuencia de UPP se asoció a la edad de manera directa, tal como se muestra en el gráfico 2.

\section{DISCUSIÓN}

Los riesgos de ulceras por presión se perciben incluso en el inicio de la cadena de atención del paciente como en el traslado en la ambulancia y el paso por emergencia donde se hace meritorio una valoración del riesgo (1).

En nuestro estudio se incluyeron 30 casos de UPP reportados por la Oficina de Inteligencia sanitaria del hospital, un caso no fue considerado en este estudio por no contar con el dato del servició donde ocurrió. Se encontró una mayor frecuencia de UPP en pacientes con estancia hospitalaria en los servicios de UCI $(41,67 \%)$.

Además, se encontró una relación directa entre la frecuencia de UPP con la edad. Respecto al género nuestro estudio mostró que el $63 \%$ de pacientes que desarrollaron UPP fueron mujeres, aunque no podemos afirmar que haya significancia estadística. Un estudio anterior de recopilación de datos de 17 estudios en UCI menciona "que los factores de riesgo más determinantes fueron edad, tiempo de estancia en UCI, diabetes, tiempo de presión arterial 
media $<60-70 \mathrm{~mm} \mathrm{Hg}$, ventilación mecánica, terapia de hemofiltración continua o diálisis intermitente, tratamiento de drogas vasoactivas, sedantes y cambios posturales, concluyendo que la aparición de las lesiones es debido a una interrelación de los factores de riesgo incrementando la probabilidad de desarrollo de las UPP" (10).

Los pacientes que ingresan a un hospital son confinados a la cama, camillas o sillas con el riesgo de desarrollar úlceras por presión, con la consecuencia de aumentar la morbilidad y deteriorar su calidad de vida, demandando mayor cuidado médico y de enfermería que en los casos de infecciones complican la salud del paciente y en algunos casos son el factor principal en el incremento de la mortalidad de estos, además del gasto financiero que esto merece que se manifiesta en billones de dólares para el tratamiento directo o indirecto de las UPP $(9,11-12)$.

La prevalencia de las UPP según un estudio en Europa es de $0 \%$ a $46 \%$ (1). Otro estudio menciona que en Brasil, la prevalencia varía entre $27 \%$ y $39,4 \%$ (8), en Inglaterra, Alemania, Suiza, Italia y Holanda la prevalencia fue $7,9 \%, 8,3 \%, 20 \%, 23 \%$ y $24,2 \%$, respectivamente (8), en Corea del Sur la prevalencia varía entre $10,5 \%$ y $45,5 \%$ (8), y en Estados Unidos las UPP es del 15\%, incrementando la hospitalización y los costos de los pacientes en un 50\% (8). En nuestro país, un estudio menciono que el $97 \%$ de pacientes hospitalizados presentan riesgo de desarrollar UPP $(13,14)$. En nuestro caso trabajamos con el número de UPP por servicios determinando que UCI, cirugía general, medicina interna y obstetricia fueron las que presentaron mayores frecuencias respecto al número de UPP mostrando una relación estadística entre las UPP y los servicios.

Lo referido anteriormente denota la importancia para el sistema hospitalario respecto que debe demandar en la prevención de las UPP en nuestro país, para no generar un deterioro significativo de la calidad de vida de los pacientes.

La limitación del estudio es que no se contó con los números totales de pacientes hospitalizados respecto al género y edad y determinar si la edad y el género fueron factores asociados a las UPP.

En conclusión, se encontró que la frecuencia de úlceras por presión se asoció con el servicio de hospitalización del paciente, siendo más frecuente en la unidad de cuidados intensivos. Más del 70\% de los casos de UPP ocurrieron en la UCI, hospitalización de cirugía, medicina y obstetricia.

\section{Agradecimiento:}

Se agradece al Dr. José Oliver Iannacone por el apoyo con los trabajos estadísticos.

\section{Declaración de financiamiento y de conflictos de intereses:}

El trabajo fue financiado por los autores, quienes declaran no tener conflictos de intereses.

\section{Contribución de autoría:}

YFL y JRJ: Participaron en la concepción del artículo, la recolección de datos, su redacción, aprobación de la versión final. JJR Participó en la recolección de datos $\mathrm{y}$ análisis de estos y aprobación final del estudio.

\section{Correspondencia:}

Jesús Rojas Jaimes

Dirección: Av. Alfredo Mendiola 5210, Los Olivos.

Lima, Perú,

Teléfono: 51993638840

Correo electrónico: jesus.rojas.jaimes@gmail.com

\section{REFERENCIAS BIBLIOGRÁFICAS}

1. Muntlin A, Engstrom M, Gunningberg L, Baath C. Heel pressure ulcer, prevention and predictors during the care delivery chain - when and where to take action? A descriptive and explorative study. Scand J Trauma Resusc Emerg Med. 2016; 24(1):1-7.

2. Norton PG, Murray M, Doupe MB, et al. Facility versus unit level reporting of quality indicators in nursing homes when performance monitoring is the goal. BMJ Open. 2014; 4(2):1-7.

3. Jiménez V, Gálvez D, de la Rosa H. Beneficio de la aplicación de la escala de Norton en pacientes graves. Unidad de Cuidados Intensivos. Hospital Militar Docente Dr. Mario Muñoz Monroy. Matanzas. Febrero-diciembre. 2009. Rev Médica Electrónica. 2010; 32(5):1-6.

4. García FP, Pancorbo PL, Soldevilla JJ, Blasco C. Escalas de valoración del riesgo de desarrollar úlceras por presión. Gerokomos. 2008; 19(3):136-44.

5. García-Fernández FP, Pancorbo-Hidalgo PL, Soldevilla-Agreda J, Rodríguez-Torres M. Valoración del riesgo de desarrollar úlceras por presión en unidades de cuidados críticos: Revisión sistemática con metaanálisis en la unidad de cuidados críticos. 
GEROKOMOS. 2013; 24(2):84-91.

6. Borghardt AT, Prado TN, Araújo TM, De Rogenski NMB, Bringuente MEDO. Evaluation of the pressure ulcers risk scales with critically ill patients: a prospective cohort study. Rev Lat Am Enfermagem. 2015; 23(1):28-35.

7. Kwong EW, Hung MS, Woo K. Improvement of pressure ulcer prevention care in private for-profit residential care homes: an action research study. BMC Geriatr. 2016; 16(1):1-14.

8. Poss J, Murphy KM, Woodbury MG, et al. Development of the interRAI Pressure Ulcer Risk Scale (PURS) for use in long-term care and home care settings. BMC Geriatr. 2010; 10(67): 1-10.

9. Meesterberends E, Halfens RJ, Heinze C, Lohrmann C, Schols JM. Pressure ulcer incidence in Dutch and German nursing homes: design of a prospective multicenter cohort study. BMC Nurs. 2011; 10(1):16.

10. Lima Serrano M, González Méndez MI, Carrasco Cebollero FM, Lima Rodriguez JS. Factores de riesgo asociados al desarrollo de úlceras por presión en unidades de cuidados intensivos de adultos: revisión sistemática. Med Intensiva. 2017; 41(6):33946.
11. Araújo TM De, Flávio M, Araújo M, Áfio J. Comparison of risk assessment scales for pressure ulcers in critically ill patients. Act Paul Enferm. 2011; 24(5):695-700.

12. Schoonhoven L, Haalboom JRE, Bousema MT, et al. Prospective cohort study of routine use of risk assessment scales for prediction of pressure ulcers. BMJ. 2002; 1 (325) 1-5.

13. Nahui A. Factores de riesgo asociados a presencia de ulceras por presión (UPP) en pacientes hospitalizados en el área de traumatología del hospital nacional Edgardo Rebagliati Martins. Tesis de bachiller para optar el título de licenciado en enfermería. Lima, Perú: Escuela Profesional de Enfermería, Universidad Privada Juan Pablo II; 2014. 106 p.

14. Acarruz-Vargas C, Tapia-Estrada D, Tito-Pacheco E, Vaiz-Bonifaz R. Valoración del riesgo de ulcera por presión según la escala de Braden en el paciente neurológico. Rev enferm Herediana. 2014; 7(1):1016.

Recibido: 20/01/2019

Aceptado: 18/06/2020 